QUARTERLY OF APPLIED MATHEMATICS

VOLUME LXVI, NUMBER 2

JUNE 2008, PAGES 303-324

S 0033-569X(08)01116-1

Article electronically published on February 8, 2008

\title{
VARIATIONAL APPROACH TO NONLINEAR GRAVITY-DRIVEN INSTABILITIES IN A MHD SETTING
}

\author{
BY
}

HYUNG JU HWANG

Department of Mathematics, Pohang University of Science and Technology, Pohang 790-784, Korea

Abstract. We establish a variational framework for nonlinear instabilities in a setting of the ideal magnetohydrodynamic (MHD) equations. We apply a variational method to unstable smooth steady states for both incompressible and compressible ideal MHD equations. The destabilizing effect of compressibility is justified along with the stabilizing effect of magnetic field lines arising in the MHD dynamics. This generalizes the result of the Rayleigh-Taylor instability for incompressible fluids in the absence of magnetic field lines; see Hwang and Guo, On the dynamical Rayleigh-Taylor instability, Arch. Rational Mech. Anal. 167 (2003), 235-253.

1. Introduction. Magnetohydrodynamic equations (MHD) serve as an important model for fluid and gas dynamics, and hydromagnetic instability is a fundamental phenomenon in nature, for instance, oceans, atmosphere, and plasma. Nevertheless, not many analytical results exist to date due to the structural complexity such as the presence of shock waves. Furthermore, nothing is known about nonlinear instabilities for the MHD equations.

The main purpose of this article is to present a variational framework in the passage from linear to nonlinear instability in a setting of the ideal MHD system and to derive the nonlinear instability around different unstable steady states for both incompressible and compressible ideal MHD fluids.

We consider the equations of the ideal magnetohydrodynamics (MHD) for inviscid flows:

$$
\begin{aligned}
\rho_{t}+\nabla \cdot(\rho V) & =0, \\
\rho\left(V_{t}+V \cdot \nabla V\right) & =(\nabla \times B) \times B-\nabla P+\rho \vec{g}, \\
B_{t} & =\nabla \times(V \times B), \\
\nabla \cdot B & =0 .
\end{aligned}
$$

Received July 1, 2006.

2000 Mathematics Subject Classification. Primary 76E30.

E-mail address: hjhwang@postech.ac.kr 
Here $\rho$ is the plasma density, $V$ is the velocity, $B$ is the magnetic field, $P$ is the plasma pressure, and $\vec{g}$ is the gravitational field. The $x$ axis is taken along the gravitational field, which is assumed to be uniform:

$$
\vec{g}=(g, 0,0) .
$$

The condition for a steady state $\left(\rho_{0}, B_{0}, P_{0}\right)$ with $V_{0} \equiv 0$ is then

$$
\frac{d}{d x}\left(P_{0}+\frac{1}{2} B_{0}^{2}\right)=\rho_{0} g .
$$

We assume that

$$
\min _{x} \rho_{0} \geq c>0
$$

The domain is $t \geq 0$ and

$$
D=\{0 \leq x \leq 2 \pi, 0 \leq y \leq 2 \pi\} .
$$

We assume periodic conditions at the boundary for $V$ and $B$. Both incompressible and compressible fluids are considered. In the incompressibility case,

$$
\nabla \cdot V=0 \text {. }
$$

In the compressibility case, $\gamma$ is an adiabatic index that relates the pressure $P$ to the density $\rho$ by

$$
\begin{aligned}
P(\rho) & =C \rho^{\gamma}, \\
\frac{\nabla P}{\rho} & =C \gamma \rho^{\gamma-2} \nabla \rho=: q(\rho) \nabla \rho .
\end{aligned}
$$

Equivalently,

$$
p_{t}+v \cdot \nabla p_{0}+\gamma p_{0} \nabla \cdot v=0
$$

We now consider a perturbation $(\sigma, v, B, p)$ around the steady state $\left(\rho_{0}, B_{0}, V_{0} \equiv 0, P_{0}\right)$ of the form:

$$
\begin{aligned}
v_{1} & =v_{1}(t, x, y), v_{2}=v_{2}(t, x, y), v_{3}=v_{3}(t, x, y), \\
B_{1} & =B_{1}(t, x, y), B_{2}=B_{2}(t, x, y), B_{3}=B_{3}(t, x, y) .
\end{aligned}
$$

Equations for perturbed quantities take the form:

$$
\begin{aligned}
\sigma_{t}+\nabla \cdot\left[\left(\rho_{0}+\sigma\right) v\right] & =0, \\
\left(\rho_{0}+\sigma\right)\left(v_{t}+v \cdot \nabla v\right) & =\left(\nabla \times B_{0}\right) \times B+(\nabla \times B) \times B_{0} \\
& +(\nabla \times B) \times B-\nabla p+\sigma \vec{g}, \\
B_{t} & =\nabla \times\left(v \times\left(B_{0}+B\right)\right), \\
\nabla \cdot B & =0 .
\end{aligned}
$$

We also obtain its linearized system:

$$
\begin{aligned}
\sigma_{t}+\nabla \cdot\left(\rho_{0} v\right) & =0, \\
\rho_{0} v_{t} & =\left(\nabla \times B_{0}\right) \times B+(\nabla \times B) \times B_{0}-\nabla p+\sigma \vec{g}, \\
B_{t} & =\nabla \times\left(v \times B_{0}\right), \\
\nabla \cdot B & =0 .
\end{aligned}
$$


In the incompressibility case, $v$ also satisfies

$$
\nabla \cdot v=0 .
$$

It is crucial to make an equivalent second-order linear system in $v$ and to use it for our variational formulation. By taking the $t$-derivative of the $v$-equation in (1.7) and plugging the $\sigma$-and the $B$-equation in (1.7) (also (1.5) in the compressibility case) into the resulting equation, we obtain the following second-order linear hyperbolic PDE in $v$ :

$$
\rho_{0} v_{t t}=(Q \cdot \nabla) B_{0}+\left(B_{0} \cdot \nabla\right) Q-\nabla\left(B_{0} \cdot Q+p_{t}\right)-\vec{g} \nabla \cdot\left(\rho_{0} v\right)=: \mathcal{L}(v),
$$

where $Q=\nabla \times\left(v \times B_{0}\right)$ and we have used (1.11).

For notational convenience,

Notation 1. For any $u$ and $v$ in $L^{2}(0,2 \pi)$,

$$
\langle u, v\rangle=\int_{0}^{2 \pi} u \cdot v d x,(u, v)=\int_{0}^{2 \pi} \rho_{0} u \cdot v d x .
$$

For any $u$ and $v$ in $L^{2}(D)$,

$$
\langle u, v\rangle=\iint_{D} u \cdot v d x d y,(u, v)=\iint_{D} \rho_{0} u \cdot v d x d y
$$

Notation 2. $\|f\|=(f, f)^{1 / 2},\|f\|_{s}=\left(\sum_{\alpha}\left\|\partial^{\alpha} f\right\|^{2}\right)^{1 / 2}$, where $\alpha$ is a multi-index with $|\alpha| \leq s$.

We make the following variational formulation and obtain a discrete set of eigenvalues for the linear operator (1.8). For each wave number $k \in \mathbb{N}$,

$$
\lambda_{k}^{2}=\sup _{\substack{u=\left(u_{1}, u_{2}\right) \\ u_{1} \in H^{\mu}(0,2 \pi) \\ u_{2} \in H^{\mu-1}(0,2 \pi)}} \frac{\langle\mathcal{L}(u), u\rangle}{(u, u)},
$$

where $\partial_{y}$ is replaced by the multiplication with $k$ in $\mathcal{L}(u)$ and $\mu=1$ or 2 . Notice that the right-hand side of (1.9) is a function of $k$.

In the incompressibility case, $u_{2}$ is replaced by $-\frac{1}{k} u_{1 x}$ from a divergence-free condition for the normal modes and (1.9) reduces to a variational problem for $u_{1}$ alone. On the other hand, the compressibility case may not be simplified to a formula for $u_{1}$ alone.

The key step of this article is to show that the discrete set $\left\{\lambda_{k}\right\}_{k \in \mathbb{N}}$ of the eigenvalues characterizes the spectral radius of the whole linearized operator by taking the limit as $k \rightarrow \infty$ to obtain the bounded least upper bound $\Lambda>0$. Let

$$
\lim _{k} \lambda_{k}^{2}=\Lambda^{2}=\sup _{v \in H^{\mu-1}(D)} \frac{\iint_{D} H\left(v, v_{x}\right) d x d y}{(v, v)},
$$

where the coefficients of $H$ depend on $\rho_{0}, p_{0}, B_{0}$. Then we obtain the following theorem: Theorem 1. Let $(\sigma, v, B)$ be a solution to (1.7) and let $\Lambda^{2}>0$ be a real number; then we have

$$
\|\sigma(t), v(t), B(t)\|_{s} \leq C e^{\Lambda t}\|\sigma(0), v(0), B(0)\|_{s+2},
$$

where $C=C\left(\Lambda, \rho_{0}, s\right)$ and $\Lambda>0$. 
Then one can locate a dominant eigenvalue and pass to nonlinear instability.

We establish the following dynamical instability for the fully nonlinear MHD system around different steady states with $\Lambda^{2}>0$ real as in Section 4:

Theorem 2. Steady states $\left(\rho_{0}, \overrightarrow{0}, B_{0}\right)$ of (1.1) in (1.2) with $\Lambda^{2}>0$ real are nonlinearly unstable. For any integer $s \geq 0$ large, there exists $\varepsilon_{0}>0$ such that for any small $\delta>0$, there exists a family of classical solutions $\left(\rho^{\delta}(t, x, y), V^{\delta}(t, x, y), B^{\delta}(t, x, y)\right)$ to (1.1) such that

$$
\left\|\rho^{\delta}(0, \cdot)-\rho_{0}(\cdot)\right\|_{H^{s}(D)}+\left\|V^{\delta}(0, \cdot)\right\|_{H^{s}(D)}+\left\|B^{\delta}(0, \cdot)-B_{0}(\cdot)\right\|_{H^{s}(D)} \leq \delta
$$

but for $T^{\delta}=O(|\ln \delta|)$,

$$
\sup _{0 \leq t \leq T^{\delta}}\left\{\left\|\rho^{\delta}(t, \cdot)-\rho_{0}(\cdot)\right\|_{L^{1}(D)}+\left\|V^{\delta}(t, \cdot)\right\|_{L^{1}(D)}+\left\|B^{\delta}(t, \cdot)-B_{0}(\cdot)\right\|_{L^{1}(D)}\right\} \geq \varepsilon_{0} .
$$

REMARK 1. This formally indicates the Kruskal-Schwarzschild instability for incompressible ideal MHD fluids by letting $\gamma \rightarrow \infty$.

REMARK 2. The instability time $T^{\delta}$ occurs before the possible blow-up time, which is shown in the proof.

REMARK 3. We measure instability in $L^{1}$.

The Rayleigh-Taylor instability is known as a gravity-driven instability in fluids when a heavy fluid is on top of a light one. Linear instability for an incompressible fluid was first introduced by Rayleigh in 1883 [18. Study on linear instability for the incompressible ideal MHD system in the presence of magnetic field lines is found in [16, 4 for instance, which include the classical Kruskal-Schwarzschild instability in the presence of a magnetic field orthogonal to the gravitational force field. In this case, the instability criterion (4.4) and the growth rates (4.2), (4.3) turn out to be the same as the ones in the RayleighTaylor instability [10] without the effect of magnetic field lines on the instability. On the other hand, when we consider a magnetic field parallel to the direction of the gravitational force, a stabilizing effect of magnetic field lines appears as in (4.13) and (4.14). A condition for linear instability for a compressible fluid in the absence of a magnetic field was first derived by Schwarzschild in [19, and since been studied by many physicists for a certain class of steady states; see [4, [5], 7], [1], for instance. The full consideration of gravity, magnetic field lines, and compressibility has also been taken for the linear instability (see, for instance, [1], 2], 17]).

Despite extensive research and interest in MHD systems from the physical point of view, little has been done from a mathematical perspective. In addition, the passage from linear to nonlinear instability in a conservative PDE system is quite difficult because of the following two obstacles: (1) presence of a continuum linear spectrum and (2) severely unbounded high-order terms in PDE systems. No systematic framework is available for this problem, although there have been some results for specific physical systems; see, for example, 9], 3, 10. A variational approach was first introduced by Guo and Hwang [10] in the context of the dynamical Rayleigh-Taylor instability for incompressible Euler fluids. However, it is not obvious whether it can be extended to MHD instabilities for compressible as well as incompressible fluids since MHD has a complicated structure in addition to analytical difficulties coming from the compressibility. 
A crucial point is whether and how to locate a dominant eigenvalue in the complex linear spectrum of the linear MHD system. We use a MHD energy principle in order to estimate a sharp spectral radius and make extensive use of the variational structure of the linearized MHD system in order to get precise and optimal estimates. The radius (1.10) of the spectrum of the MHD system is obtained as the least upper bound for the discrete set of the eigenvalues (1.9) of normal growing modes, and this method is explicit and constructive. We consider various magnetic fields as steady states that give rise to different outcomes in the growth rates (4.3), (4.9), (4.14), (4.15) of the instabilities and different ranges of admissible density profiles. We justify stabilizing and destabilizing effects of magnetic field lines and compressibility. Furthermore, the incompressibility case can also be viewed as a limit case of the compressibility case as $\gamma \rightarrow \infty$.

The article is organized as follows. We formulate the variational problems (1.9) in Section 2 and show the existence of smooth maximizers satisfying the corresponding Sturm-Liouville equations. We then derive a sharp growth rate $\Lambda>0$ for the whole linear MHD system in Section 3 using Theorem 1. In Section 4, we give different examples of steady states that result in different growth rates, Sturm-Liouville equations, and different admissibility for density profiles for the instability. For instance, if a steady magnetic field is parallel to the gravitational field, we have the fourth-order SturmLiouville equation (4.12). The compressibility case (4.8) is more complicated, and its variational problem should be carefully treated.

In Section 5, we construct approximate solutions and give energy estimates for the full MHD system. Finally, we present the nonlinear instability for the ideal MHD system around the steady states in Section 6 .

We present some useful vector identities which are used throughout the paper. For any two vectors $\vec{a}$ and $\vec{b}$, we have

$$
\begin{aligned}
& \nabla \times(\vec{a} \times \vec{b})=\vec{a}(\nabla \cdot \vec{b})+(\vec{b} \cdot \nabla) \vec{a}-\vec{b}(\nabla \cdot \vec{a})-(\vec{a} \cdot \nabla) \vec{b}, \\
& \nabla(\vec{a} \cdot \vec{b})=(\vec{a} \cdot \nabla) \vec{b}+(\vec{b} \cdot \nabla) \vec{a}+\vec{a} \times(\nabla \times \vec{b})+\vec{b} \times(\nabla \times \vec{a}) .
\end{aligned}
$$

2. General variational framework. We consider the following steady magnetic fields:

(Case $B_{0} \perp \vec{g}$ )

$$
B_{0}=\left(0,0, B_{0}(x)\right)
$$

$\left(\right.$ Case $\left.B_{0} \| \vec{g}\right)$

$$
B_{0}=\left(B_{0}, 0,0\right) \text {. }
$$

In the case of $B_{0} \| \vec{g}$, we assume $v_{3}=B_{3}=0$. By integration by parts, using (1.7), (1.5), (1.2), and completing the square with respect to $\nabla \cdot v$, we obtain the following decomposition for $\langle\mathcal{L}(v), v\rangle$ : for any $v=\left(v_{1}, v_{2}, v_{3}\right)$,

$$
\begin{aligned}
\langle\mathcal{L}(v), v\rangle & =\iint_{D} \mathcal{L}(v) \cdot v d x d y \\
& =\iint_{D}\left[-F\left(v_{2 x}\right)-G\left(\nabla \cdot v, v_{1}, v_{1 x}\right)+H\left(v_{1}, v_{1 x}\right)\right] d x d y .
\end{aligned}
$$

We state some important properties that are satisfied by the above functional: 
(1) $\mathcal{L}$ is variational; i.e., for any $u$ and $v$ in $H^{1}(D)$,

$$
\langle\mathcal{L}(u), v\rangle=\langle u, \mathcal{L}(v)\rangle .
$$

(2) $\langle\mathcal{L}(v), v\rangle$ is concave with respect to $v_{1 x}, v_{2 x}, v_{2}$ respectively.

(3) $\langle\mathcal{L}(v), v\rangle \rightarrow-\infty$ as $v_{1 x} \rightarrow \infty$ and $\langle\mathcal{L}(v), v\rangle \rightarrow-\infty$ as $v_{2 x} \rightarrow \infty$ if $F \neq 0$.

(4) $F>0, G>0, F, G, H$ are all quadratic, and $H(0)=0$ when $H=H\left(v_{1}\right)$ alone.

REMARK 4. $G=0$ in the incompressibility case.

REmark 5. For the case $B_{0} \perp \vec{g}$, we have $F=0, H=H\left(v_{1}\right)$, and $G=G\left(\nabla \cdot v, v_{1}\right)$, while we have $F=F\left(v_{2 x}\right), H=H\left(v_{1}, v_{1 x}\right), G=G\left(\nabla \cdot v, v_{1}, v_{1 x}\right)$ in the case $B_{0} \| \vec{g}$ as we will see in Section 4.

We will show that for any fixed wave number $k \in \mathbb{N}$, the corresponding eigenvalue $\lambda_{k}>0$ for the linearized MHD system takes the variational formulation closely related to the above variational structure. A normal mode takes the form:

$$
\begin{aligned}
& v_{1}(t, x, y)=\tilde{v}_{1}(x) \cos (k y) \exp \left(\lambda_{k} t\right), \\
& v_{2}(t, x, y)=\tilde{v}_{2}(x) \sin (k y) \exp \left(\lambda_{k} t\right), \\
& v_{3}(t, x, y)=\tilde{v}_{3}(x) \cos (k y) \exp \left(\lambda_{k} t\right),
\end{aligned}
$$

where $k$ is a wave number. Substituting (2.2) into (1.8) yields the following second-order ODE for $\tilde{v}$ :

$$
\lambda_{k}^{2} \rho_{0}\left(\tilde{v}_{1}, \tilde{v}_{2}, \tilde{v}_{3}\right)=\mathcal{L}\left(\tilde{v}_{1}, \tilde{v}_{2}, \tilde{v}_{3}\right) .
$$

Note that $\mathcal{L}_{3}\left(\tilde{v}_{1}, \tilde{v}_{2}, \tilde{v}_{3}\right)=0$ and thus $\tilde{v}_{3}=0$. We now make the following variational formulation:

$$
\lambda_{k}^{2}=\sup _{\substack{u=\left(u_{1}, u_{2}\right) \\ u_{1} \in H^{\mu}(0,2 \pi) \\ u_{2} \in H^{\mu-1}(0,2 \pi)}} \frac{\langle\mathcal{L}(u), u\rangle}{(u, u)}
$$

where $\mu=1$ or $2, \partial_{y}$ is replaced by the multiplication with $k$ in $\mathcal{L}(u)$, and the integrations in (2.4) are over $(0,2 \pi)$ with respect to $x$.

Incompressibility case: we use the divergence-free condition to reduce to

$$
\lambda_{k}^{2}=\sup _{u \in H^{\mu}(0,2 \pi)} \frac{\int_{0}^{2 \pi}\left[-F\left(-\frac{u_{x x}}{k}\right)+H\left(u, u_{x}\right)\right] d x}{\int_{0}^{2 \pi}\left[u^{2}+\frac{u_{x}^{2}}{k^{2}}\right] d x},
$$

where $\mu=1$ or 2 .

Compressibility case:

$$
\lambda_{k}^{2}=\sup _{\substack{u_{1} \in H^{\mu}(0,2 \pi) \\ u_{2} \in H^{\mu-1}(0,2 \pi)}} \frac{\int_{0}^{2 \pi}\left[-F\left(u_{2 x}\right)-G\left(u_{1 x}+k u_{2}, u_{1}, u_{1 x}\right)+H\left(u_{1}, u_{1 x}\right)\right] d x}{\int_{0}^{2 \pi}\left[u_{1}^{2}+u_{2}^{2}\right] d x},
$$

where $\mu=1$ or 2 .

Let

$$
\Lambda^{2}=\sup _{v \in H^{\mu-1}(D)} \frac{\iint_{D} H\left(v, v_{x}\right) d x d y}{(v, v)},
$$

where $\mu=1$ or 2 . Then we show that $\Lambda^{2}$ is the least upper bound for $\left\{\lambda_{k}^{2}\right\}_{k \in N}$. 
LEMMA 1.

$$
\lim _{k \rightarrow \infty} \lambda_{k}^{2}=\Lambda^{2}
$$

Proof. Since $F(0)=0$, it is easy to see that the lemma is true for the incompressibility case by letting $k \rightarrow \infty$. We now treat the compressibility case.

Note that we have, by choosing $u_{2}=-\frac{1}{k}\left(a u_{1}+b u_{1 x}\right)$,

$$
\begin{aligned}
\lambda_{k}^{2} & =\sup _{\substack{u=\left(u_{1}, u_{2}\right) \\
u_{1} \in H^{\mu}(0,2 \pi) \\
u_{2} \in H^{\mu-1}(0,2 \pi)}} \frac{\langle\mathcal{L}(u), u\rangle}{(u, u)} \\
& \geq \sup _{u_{1} \in H^{\mu}(0,2 \pi)} \frac{\int_{0}^{2 \pi}\left[-F\left(-\frac{1}{k}\left(a u_{1}+b u_{1 x}\right)_{x}\right)+H\left(u_{1}, u_{1 x}\right)\right] d x}{\int_{0}^{2 \pi} \rho_{0}\left[u_{1}^{2}+\frac{1}{k^{2}}\left(a u_{1}+b u_{1 x}\right)^{2}\right] d x} .
\end{aligned}
$$

Thus we have the following inequality:

$$
\begin{aligned}
& \sup _{u_{1} \in H^{\mu}(0,2 \pi)} \frac{\int_{0}^{2 \pi}\left[-F\left(-\frac{1}{k}\left(a u_{1}+b u_{1 x}\right)_{x}\right)+H\left(u_{1}, u_{1 x}\right)\right] d x}{\int_{0}^{2 \pi} \rho_{0}\left[u_{1}^{2}+\frac{1}{k^{2}}\left(a u_{1}+b u_{1 x}\right)^{2}\right] d x} \leq \lambda_{k}^{2} \\
\leq & \sup _{\substack{u_{1} \in H^{\mu}(0,2 \pi) \\
u_{2} \in H^{\mu-1}(0,2 \pi)}} \frac{\int_{0}^{2 \pi}\left[-F\left(u_{2 x}\right)-G\left(u_{1 x}+k u_{2}, u_{1}, u_{1 x}\right)\right] d x}{\int_{0}^{2 \pi} \rho_{0}\left[u_{1}^{2}+u_{2}^{2}\right] d x} \\
+\sup _{\substack{u_{1} \in H^{\mu}(0,2 \pi) \\
u_{2} \in H^{\mu-1}(0,2 \pi)}} & \frac{\int_{0}^{2 \pi} H\left(u_{1}, u_{1 x}\right) d x}{\int_{0}^{2 \pi} \rho_{0}\left[u_{1}^{2}+u_{2}^{2}\right] d x} \\
\leq & \sup _{u_{1} \in H^{\mu-1}(0,2 \pi)} \frac{\int_{0}^{2 \pi} H\left(u_{1}, u_{1 x}\right) d x}{\int_{0}^{2 \pi} \rho_{0} u_{1}^{2} d x} .
\end{aligned}
$$

Since

we have, by letting $k \rightarrow \infty$,

$$
\frac{1}{k}\left(a u_{1}+b u_{1 x}\right) \rightarrow 0 \text { as } k \rightarrow \infty \text { and } F(0)=0,
$$

$$
\lim _{k \rightarrow \infty} \lambda_{k}^{2}=\sup _{u_{1} \in H^{\mu-1}(0,2 \pi)} \frac{\int_{0}^{2 \pi} H\left(u_{1}, u_{1 x}\right) d x}{\int_{0}^{2 \pi} \rho_{0} u_{1}^{2} d x},
$$

and

$$
\lim _{k \rightarrow \infty} \lambda_{k}^{2}=\sup _{u_{1} \in H^{\mu-1}(D)} \frac{\iint_{D} H\left(u_{1}, u_{1 x}\right) d x d y}{\iint_{D} \rho_{0} u_{1}^{2} d x d y} .
$$

Thus the proof is complete.

We now show the existence of a maximizer for the variational problem (2.4). Assume that

$$
(u, u)=\int_{0}^{2 \pi} \rho_{0}\left[u_{1}^{2}+u_{2}^{2}\right] d x=1
$$

For $k \in \mathbb{N}$ fixed, let

$$
\lambda_{k}^{2}=\sup _{\substack{u_{1} \in H^{\mu}(0,2 \pi) \\ u_{2} \in H^{\mu-1}(0,2 \pi)}}\langle\mathcal{L}(u), u\rangle .
$$


Lemma 2. For any fixed $k \in \mathbb{N}$, there exists a smooth maximizer for the variational problem (2.7) with the constraint (2.6).

Proof. Let $\left\{u_{1}^{n}, u_{2}^{n}\right\}$ be a maximizing sequence with the constraint (2.6). Then $u_{1}^{n}$ and $u_{2}^{n}$ converge weakly in $L^{2}(0,2 \pi)$ to $u_{1}^{0}$ and $u_{2}^{0}$, respectively, and we have

$$
\left\langle\mathcal{L}\left(u^{n}\right), u^{n}\right\rangle \rightarrow \lambda_{k}^{2}
$$

Case 1. $B_{0} \perp \vec{g}$, where $F=0, H=H\left(u_{1}\right)$ :

Since $\langle\mathcal{L}(u), u\rangle \rightarrow-\infty$ as $u_{1 x} \rightarrow \infty$ (Property 3 of the functional), $u_{1 x}$ is bounded in $L^{2}(0,2 \pi)$ uniformly in $n$. Thus there exists a weak limit $\left\{u_{1}^{0}, u_{2}^{0}\right\}$ such that

$$
\begin{aligned}
u_{1 x}^{n} & \rightarrow u_{1 x}^{0} \text { weakly in } L^{2}(0,2 \pi), u_{2 x}^{n} \rightarrow u_{2}^{0} \text { weakly in } L^{2}(0,2 \pi), \\
u_{1}^{n} & \rightarrow u_{1}^{0} \text { strongly in } L^{2}(0,2 \pi) \text { and } u_{1}^{0} \in H^{1}(0,2 \pi) .
\end{aligned}
$$

Next we show that $\left\{u_{1}^{0}, u_{2}^{0}\right\}$ is a maximizer and satisfies the constraint (2.6). Since $\left\{u_{1}^{0}, u_{2}^{0}\right\}$ is a weak limit of $\left\{u_{1}^{n}, u_{2}^{n}\right\},\left(u^{0}, u^{0}\right)=\int_{0}^{2 \pi} \rho_{0}\left[\left(u_{1}^{0}\right)^{2}+\left(u_{2}^{0}\right)^{2}\right] d x \leq 1$ by the lower semi-continuity of $L^{2}$ :

$$
\begin{aligned}
& \int_{0}^{2 \pi} \rho_{0}\left[\left(u_{1}^{n}\right)^{2}+\left(u_{2}^{n}\right)^{2}\right] d x-\int_{0}^{2 \pi} \rho_{0}\left[\left(u_{1}^{0}\right)^{2}+\left(u_{2}^{0}\right)^{2}\right] d x \\
= & \int_{0}^{2 \pi} 2 \rho_{0}\left[u_{1}^{0}\left(u_{1}^{n}-u_{1}^{0}\right)+u_{2}^{0}\left(u_{2}^{n}-u_{2}^{0}\right)\right] d x \\
& +\int_{0}^{2 \pi} \rho_{0}\left[\left(u_{1}^{n}-u_{1}^{0}\right)^{2}+\left(u_{2}^{n}-u_{2}^{0}\right)^{2}\right] \\
\geq & \int_{0}^{2 \pi} 2 \rho_{0}\left[u_{1}^{0}\left(u_{1}^{n}-u_{1}^{0}\right)+u_{2}^{0}\left(u_{2}^{n}-u_{2}^{0}\right)\right] d x \rightarrow 0 \text { as } n \rightarrow 0,
\end{aligned}
$$

since $\left\{u_{1}^{0}, u_{2}^{0}\right\}$ is a weak limit of $\left\{u_{1}^{n}, u_{2}^{n}\right\}$ in $L^{2}(0,2 \pi)$. We use the concavity of the functional $\langle\mathcal{L}(u), u\rangle$ with respect to $u_{1 x}, u_{2 x}, u_{2}$ (Property 2 of the functional) and the strong convergence of $u_{1}^{n}$ to $u_{1}^{0}$ in $L^{2}(0,2 \pi)$ to deduce

$$
\left\langle\mathcal{L}\left(u^{0}\right), u^{0}\right\rangle \geq \lambda_{k}^{2}
$$

Let

$$
J\left(u_{2 x}, u_{1 x}, u_{2}, u_{1}\right)=\langle\mathcal{L}(u), u\rangle .
$$

Then by the concavity of this functional $J$, the strong convergence of $u_{1}^{n}$ to $u_{1}^{0}$, and the weak convergence of $u_{1 x}^{n}, u_{2}^{n}, u_{1}^{n}$ to $u_{1 x}^{0}, u_{2}^{0}, u_{1}^{0}$, respectively, we have

$$
\begin{aligned}
& J\left(u_{2 x}^{n}, u_{1 x}^{n}, u_{2}^{n}, u_{1}^{n}\right)-J\left(u_{2 x}^{0}, u_{1 x}^{0}, u_{2}^{0}, u_{1}^{0}\right) \\
= & J\left(u_{2 x}^{n}, u_{1 x}^{n}, u_{2}^{n}, u_{1}^{n}\right)-J\left(u_{2 x}^{n}, u_{1 x}^{n}, u_{2}^{n}, u_{1}^{0}\right)+J\left(u_{2 x}^{n}, u_{1 x}^{n}, u_{2}^{n}, u_{1}^{0}\right)-J\left(u_{2 x}^{0}, u_{1 x}^{0}, u_{2}^{0}, u_{1}^{0}\right) \\
\leq & J\left(u_{2 x}^{n}, u_{1 x}^{n}, u_{2}^{n}, u_{1}^{n}\right)-J\left(u_{2 x}^{n}, u_{1 x}^{n}, u_{2}^{n}, u_{1}^{0}\right)+\nabla J\left(u_{2 x}^{0}, u_{1 x}^{0}, u_{2}^{0}, u_{1}^{0}\right) \\
& \cdot\left(u_{2 x}^{n}-u_{2 x}^{0}, u_{1 x}^{n}-u_{1 x}^{0}, u_{2}^{n}-u_{2}^{0}, 0\right)
\end{aligned}
$$$$
\rightarrow 0 \text { as } n \rightarrow \infty \text {. }
$$ 
Thus we obtain (2.9). Notice that $u^{0}=\left\{u_{1}^{0}, u_{2}^{0}\right\} \neq\{0,0\}$ a.e. since, by the strong convergence of $u_{1}^{n}$ to $u_{1}^{0}$ and by (2.8), we have

$$
\int_{0}^{2 \pi} H\left(u_{1}^{0}\right) d x=\lim _{n \rightarrow \infty} \int_{0}^{2 \pi} H\left(u_{1}^{n}\right) d x \geq \lambda_{k}^{2}>0,
$$

where we have used Property 4 of the functional. Suppose that $\left(u^{0}, u^{0}\right)=\alpha^{2}<1$ and let $\left(\tilde{u}_{1}^{0}, \tilde{u}_{2}^{0}\right)=\frac{1}{\alpha}\left(u_{1}^{0}, u_{2}^{0}\right)$ so that $\left(\tilde{u}^{0}, \tilde{u}^{0}\right)=1$. Then the above argument (2.9) yields

$$
\left\langle\mathcal{L}\left(\tilde{u}^{0}\right), \tilde{u}^{0}\right\rangle \geq \frac{\lambda_{k}^{2}}{\alpha}>\lambda_{k}^{2},
$$

leading to a contradiction. Thus $\left\{u_{1}^{0}, u_{2}^{0}\right\}$ is a maximizer satisfying the constraint (2.6).

Case 2. $B_{0} \| \vec{g}$, where $F\left(u_{2 x}\right)=B_{0}^{2} u_{2 x}^{2}$ :

In this case, by Property 3 of the functional, $u_{2 x}^{n}$ is also bounded in $L^{2}(0,2 \pi)$ uniformly in $n$ and thus both $u_{1}^{n}$ and $u_{2}^{n}$ converge strongly in $L^{2}(0,2 \pi)$ to $u_{1}^{0}$ and $u_{2}^{0}$ respectively. Then we have

$$
\left(u^{0}, u^{0}\right)=\int_{0}^{2 \pi} \rho_{0}\left[\left(u_{1}^{0}\right)^{2}+\left(u_{2}^{0}\right)^{2}\right] d x=1
$$

In a similar manner, we obtain (2.9) and hence $u^{0}$ is a maximizer.

We finally show that such a maximizer satisfies the generalized Sturm-Liouville equation (2.3) for both cases. For $\tau \in \mathbb{R}$ and $w=\left\{w_{1}, w_{2}\right\} \in H^{\mu}(0,2 \pi) \times H^{\mu-1}(0,2 \pi)$, define $u(\tau)=u^{0}+\tau w$. Then we have, by (4.8),

$$
\langle\mathcal{L}(u(\tau)), u(\tau)\rangle \leq \lambda_{k}^{2}(u(\tau), u(\tau)) .
$$

Set

$$
I(\tau)=\langle\mathcal{L}(u(\tau)), u(\tau)\rangle-\lambda_{k}^{2}(u(\tau), u(\tau)),
$$

then we have $I(\tau) \leq 0$ for all $\tau \in \mathbb{R}$ and $I(0)=0$. This implies

$$
\begin{aligned}
I^{\prime}(0) & =\left\langle\mathcal{L}(w), u^{0}\right\rangle+\left\langle\mathcal{L}\left(u^{0}\right), w\right\rangle-2 \lambda_{k}^{2}\left(u^{0}, w\right) \\
& =2\left\langle\mathcal{L}\left(u^{0}\right)-\lambda_{k}^{2} \rho_{0} u^{0}, w\right\rangle=0 \text { for all } w,
\end{aligned}
$$

since $\mathcal{L}$ is variational (Property 1 of the functional). Thus $u^{0}$ satisfies a normal mode equation, i.e.,

$$
\lambda_{k}^{2} \rho_{0} u^{0}=\mathcal{L}\left(u^{0}\right) .
$$

Since $\rho_{0}, p_{0}$, and $B_{0}$ are smooth, $u^{0}$ is also smooth. This completes the proof.

3. Linear growth rate $\Lambda$. In this section, we show that $\Lambda$ is the optimal growth rate for the linearized system and it serves as the spectral radius of the linear operator. We state the global existence of solutions to the linearized MHD system, which can be obtained by a straightforward method.

Lemma 3. For an integer $s \geq 1$ and $\left(\sigma_{0}, v_{0}, B_{0}\right) \in\left(H^{s+2}(D)\right)^{7}$, there exists a global-intime solution $(\sigma, v, B) \in C\left([0, T] ;\left(H^{s}(D)\right)^{7}\right)$ to the linearized MHD system (1.7).

Let $(\sigma, v)$ be a solution to (1.7) and let $\Lambda^{2}>0$ be a real number; then we have 
Theorem 3. For an integer $s \geq 1$ and $\left(\sigma_{0}, v_{0}, B_{0}\right) \in\left(H^{s+2}(D)\right)^{7}$, we have

$$
\|\sigma(t), v(t), B(t)\|_{s} \leq C e^{\Lambda t}\|\sigma(0), v(0), B(0)\|_{s+2},
$$

where $C=C\left(\Lambda, \rho_{0}, s\right)$ and $\Lambda>0$.

Proof. We prove the theorem by induction on $\bar{s}$, the number of the $x$-derivatives. We first treat the case $\bar{s}=0$. We multiply (4.6) by $v_{t}$ and integrate over $x$ and $y$ to get

$$
\frac{d}{d t}\left(v_{t}, v_{t}\right)=\frac{d}{d t}\langle\mathcal{L}(v), v\rangle,
$$

since $\mathcal{L}$ is variational. Notice that using (2.5) yields

$$
\iint_{D} H\left(v_{1}, v_{1 x}\right) d x d y \leq \Lambda^{2}\left(v_{1}, v_{1}\right) \leq \Lambda^{2}(v, v) .
$$

By integrating (3.1) over time and by (2.1), we obtain

$$
\begin{aligned}
& \left(v_{t}, v_{t}\right)+\iint_{D}\left[F\left(v_{2 x}\right)+G\left(\nabla \cdot v, v_{1}, v_{1 x}\right)\right] d x d y \\
\leq & \iint_{D} H\left(v_{1}, v_{1 x}\right) d x d y+\|\sigma(0), v(0), B(0)\|_{1}^{2} \\
\leq & \Lambda^{2}(v, v)+\|\sigma(0), v(0), B(0)\|_{1}^{2},
\end{aligned}
$$

and

$$
\frac{d}{d t}\|v\| \leq\left\|v_{t}\right\| \leq\|\sigma(0), v(0), B(0)\|_{1}+\Lambda\|v\| .
$$

Thus we have

$$
\|v\| \leq C e^{\Lambda t},
$$

where $C=\|\sigma(0), v(0), B(0)\|_{1}$. From now on, we will use the universal constant $C$ which varies from line to line. Notice that these estimates apply exactly to the $t$ - and the $y$-derivatives of any order of $v$ and $\sigma$ since the variational structure of (1.8) is not destroyed by taking the $t$ - and the $y$-derivatives.

CASE 1. $B_{0} \perp \vec{g}$ where $F=0, G=\left(\gamma p_{0}+B_{0}^{2}\right)\left(\nabla \cdot v+\frac{g \rho_{0}}{\gamma p_{0}+B_{0}^{2}} v_{1}\right)^{2}(G=0$ in the incompressibility case), $H=H\left(v_{1}\right),(Q \cdot \nabla) B_{0}+\left(B_{0} \cdot \nabla\right) Q=0$ :

By (3.2) and (1.7), we have

$$
\|\nabla \cdot v\| \leq C e^{\Lambda t},\|\sigma\| \leq C e^{\Lambda t},\|B\| \leq C e^{\Lambda t} .
$$

Next, we consider the case $\bar{s}=1$. By taking the curl of the $v$-equation of (1.7), we have

$$
\rho_{0}\left(\omega_{t t}\right)=-\nabla \rho_{0} \times v_{t t}-g \sigma_{t y} \hat{k},
$$

where $\hat{k}$ is the unit vector in the $z$-direction and $\omega=\nabla \times v$. Since $\sigma_{t y}$ and $v_{t t}$ have no $x$-derivatives, $B_{1 x}=-B_{2 y}$, and therefore possess the growth rate $\Lambda$ as in the previous step, we have

$$
\|\omega\| \leq C e^{\Lambda t}
$$


where $C=\|\sigma(0), v(0), B(0)\|_{3}$. Thanks to the identity $\Delta \zeta=-\nabla \times(\nabla \times \zeta)+\nabla(\nabla \cdot \zeta)$ for any $\zeta=\left(\zeta_{1}, \zeta_{2}\right)$, we conclude that all of the first derivatives of $v$ have the same growth rate $\Lambda$. Now for $\nabla \sigma$, we use the vector identity (1.11) to obtain

$$
B_{t}=\nabla\left(v \times B_{0}\right)=-B_{0 x} v_{1} \hat{k}-\hat{k} B_{0} \nabla \cdot v .
$$

Plugging (3.4) and (1.5) into (1.8) yields

$$
\rho_{0} v_{t t}=\nabla\left(B_{0} B_{0 x} v_{1}\right)+\nabla\left(\left[\gamma p_{0}+B_{0}^{2}\right] \nabla \cdot v\right)+\sigma_{t} \vec{g} .
$$

By the induction hypotheses and the $\sigma$ - and $B$-equations in (1.7), we deduce

$$
\|\nabla(\nabla \cdot v)\| \leq C e^{\Lambda t},\|\nabla \sigma\| \leq C e^{\Lambda t},\|\nabla B\| \leq C e^{\Lambda t} .
$$

For higher derivatives when $\bar{s} \geq 2$, we use the induction hypotheses. Suppose all of the derivatives of order $<\bar{s}$ have the growth rate $\Lambda$. Let $\alpha$ be a multi index whose order of the $x$-derivative is $\bar{s}-1$. Then, by taking $\partial_{\alpha}$ of the curl of (4.6), $\operatorname{curl}\left(\partial_{\alpha} v\right)$ satisfies

$$
\rho_{0}\left(\partial_{\alpha} \omega\right)_{t t}=-\partial_{a}\left(\nabla \times\left(\rho_{0} v\right)\right)_{t t}+\rho_{0}\left(\partial_{\alpha} \omega\right)_{t t}-g \partial_{\alpha} \sigma_{t y} \hat{k} .
$$

The right-hand side of (3.6) contains derivatives of $v$ and $\sigma$ whose $x$-order $<\bar{s}$. Clearly the $x$-order of $\partial_{\alpha} \sigma_{t y}$ is $\bar{s}-1$. The first and the second terms on the right-hand side together are of the form

$$
\sum_{\substack{|\beta| \geq 1 \\ \beta+\gamma=\alpha}} C_{\beta, \gamma} \nabla \times\left[\left(\partial_{\beta} \rho_{0}\right)\left(\partial_{\gamma} v_{t t}\right)\right] .
$$

Notice that only purely $x$-derivatives $\partial_{\beta} \rho_{0}$ with $|\beta| \geq 1$ in (3.7) remain nonzero since $\rho_{0}(x)$ depends only on $x$. Then (3.6) yields

$$
\left\|\nabla \times \partial_{a} v\right\| \leq C e^{\Lambda t}
$$

For $\nabla \cdot \partial_{\alpha} v$, we take $\partial_{\beta}$ of the first component of (3.5) with $\partial_{\beta} \partial_{x}=\partial_{\alpha}$ to get

$$
\partial_{\beta}\left(\rho_{0} v_{1 t t}\right)=\partial_{\alpha}\left(B_{0} B_{0 x} v_{1}\right)+\partial_{\alpha}\left(\left[\gamma p_{0}+B_{0}^{2}\right] \nabla \cdot v\right)+\partial_{\beta} \sigma_{t} g .
$$

Using the induction hypotheses as for the case $\bar{s}=1$, we deduce

$$
\|v\|_{\bar{s}} \leq C e^{\Lambda t},\|\sigma\|_{\bar{s}} \leq C e^{\Lambda t},\|B\|_{\bar{s}} \leq C e^{\Lambda t},
$$

where $C=\|\sigma(0), v(0), B(0)\|_{\bar{s}+2}$. In the incompressibility case, we use (3.3) together with the $\nabla \cdot v=0$ to deduce the lemma.

CASE 2. $B_{0} \| \vec{g}$ and incompressibility case when $F=B_{0}^{2} v_{2 x}^{2}, G=0, H=H\left(v_{1}, v_{1 x}\right)$ :

From (3.2), $\operatorname{div} v=0$, and using the induction hypotheses, we obtain

$$
\left\|v_{2 x}\right\| \leq C e^{\Lambda t},\left\|v_{1 x}\right\| \leq C e^{\Lambda t} .
$$

In this case, using (1.11) yields $Q=B_{0} v_{x}$ and $B_{0} \cdot Q=B_{0}^{2} v_{1 x}$. Then we use (1.8) to get

$$
\rho_{0} v_{t t}=B_{0}^{2} v_{x x}-\nabla\left(B_{0} \cdot Q+p_{t}\right)+\sigma_{t} \vec{g} .
$$

Taking the curl of (3.8) yields

$$
\rho_{0} \omega_{t t}=B_{0}^{2} \omega_{x x}-\nabla \rho_{0} \times v_{t t}-g \sigma_{t y} \hat{k}
$$


Using the induction hypotheses and $\operatorname{div} v=0$, we can deduce the lemma. Therefore the proof is complete.

4. Examples. In this section, we present examples of steady states that give rise to different results for instability.

(1) Magnetic field is transverse to the gravitational field $B_{0} \perp \vec{g}$ for incompressible fluids:

$$
B_{0}=\left(0,0, B_{0}(x)\right) .
$$

The linear operator $\mathcal{L}$ reads

$$
\mathcal{L}(v):=-\nabla\left(p_{t}+B_{0} \cdot Q\right)-\vec{g} \rho_{0 x} v_{1} .
$$

The Sturm-Liouville equation is

$$
\rho_{0} \lambda_{k}^{2} \tilde{v}_{1}=\frac{\lambda_{k}^{2}}{k^{2}}\left(\rho_{0} \tilde{v}_{1 x}\right)_{x}-g \rho_{0 x} \tilde{v}_{1} .
$$

The variational formulation for $\lambda_{k}^{2}$ is

$$
\lambda_{k}^{2}=\sup _{u \in H^{1}(0,2 \pi)} \frac{\int_{0}^{2 \pi} \mathcal{L}(u) \cdot u d x}{\int_{0}^{2 \pi} \rho_{0}\left[u^{2}+\frac{u_{x}^{2}}{k^{2}}\right] d x}>0,
$$

where

$$
\mathcal{L}(u) \cdot u=H(u)=-g \rho_{0 x} u^{2} .
$$

The spectral radius $\Lambda>0$ is given by

$$
\Lambda^{2}=\sup _{v \in L^{2}(D)} \frac{\iint_{D} H(v) d x d y}{\iint_{D} \rho_{0} v^{2} d x d y}>0 .
$$

The sufficient condition for instability for the case (1) is given by

$$
\rho_{0 x}\left(x_{0}\right)<0 \text { for some } x_{0} \in(0,2 \pi) .
$$

(2) Magnetic field is transverse to the gravitational field $B_{0} \perp \vec{g}$ for compressible fluids:

$$
B_{0}=\left(0,0, B_{0}(x)\right) .
$$

The linear operator $\mathcal{L}$, the Sturm-Liouville equation, and $\lambda_{k}$ read as follows:

$$
\begin{aligned}
\mathcal{L}(v)= & -\nabla\left(B_{0} \cdot Q\right)-\nabla\left(p_{0 x} v_{1}+\gamma p_{0} \nabla \cdot v\right)-\vec{g}\left(\rho_{0 x} v_{1}+\rho_{0} \nabla \cdot v\right), \\
& \lambda_{k}^{2} \rho_{0}\left(\tilde{v}_{1}, \tilde{v}_{2}, \tilde{v}_{3}\right)=\mathcal{L}\left(\tilde{v}_{1}, \tilde{v}_{2}, \tilde{v}_{3}\right),
\end{aligned}
$$

where $\mathcal{L}=\left(\mathcal{L}^{1}, \mathcal{L}^{2}, \mathcal{L}^{3}\right)$ is a linear functional in $\tilde{v}=\left(\tilde{v}_{1}, \tilde{v}_{2}, \tilde{v}_{3}\right)$ such that

$$
\begin{aligned}
& \mathcal{L}^{1}=\left(g \rho_{0} \tilde{v}_{1}+\left(\gamma p_{0}+B_{0}^{2}\right)\left(\tilde{v}_{1 x}+k \tilde{v}_{2}\right)\right)_{x}-g\left(\rho_{0 x} \tilde{v}_{1}+\rho_{0}\left(\tilde{v}_{1 x}+k \tilde{v}_{2}\right)\right), \\
& \mathcal{L}^{2}=-k\left(g \rho_{0} \tilde{v}_{1}+\left(\gamma p_{0}+B_{0}^{2}\right)\left(\tilde{v}_{1 x}+k \tilde{v}_{2}\right)\right), \\
& \mathcal{L}^{3}=0
\end{aligned}
$$


and so $\tilde{v}_{3}=0$.

$$
\lambda_{k}^{2}=\sup _{\substack{u=\left(u_{1}, u_{2}\right) \\ u_{1} \in H^{1}(0,2 \pi) \\ u_{2} \in L^{2}(0,2 \pi)}} \frac{\int_{0}^{2 \pi} \mathcal{L}(u) \cdot u d x}{\int_{0}^{2 \pi} \rho_{0}\left[u_{1}^{2}+u_{2}^{2}\right] d x}>0
$$

where

$$
\begin{aligned}
\mathcal{L}(u) \cdot u & =-G\left(u_{1}, u_{1 x}, u_{2}\right)+H\left(u_{1}\right) \\
G\left(u_{1}, u_{1 x}, u_{2}\right) & =\left(\gamma p_{0}+B_{0}^{2}\right)\left(u_{1 x}+k u_{2}+\frac{g \rho_{0}}{\gamma p_{0}+B_{0}^{2}} u_{1}\right)^{2}, \\
H\left(u_{1}\right) & =\left(\frac{g^{2} \rho_{0}^{2}}{\gamma p_{0}+B_{0}^{2}}-g \rho_{0 x}\right) u_{1}^{2} .
\end{aligned}
$$

The spectral radius $\Lambda>0$ is given by

$$
\Lambda^{2}=\sup _{v \in L^{2}(D)} \frac{\iint_{D} H(v) d x d y}{\iint_{D} \rho_{0} v^{2} d x d y}>0 .
$$

The sufficient condition for instability is given by, for some $x_{0} \in(0,2 \pi)$,

$$
\frac{g^{2} \rho_{0}^{2}}{\gamma p_{0}+B_{0}^{2}}\left(x_{0}\right)>g \rho_{0 x}\left(x_{0}\right) .
$$

Notice that density inversion is not required for instability unlike the incompressibility case. Thus, even if a light fluid is on top of a heavy fluid and magnetic field lines stabilize, such steady state flows can't sustain themselves against small initial disturbances under the effect of compressibility. It means that the destabilizing effect of compressibility dominates over the stabilizing effect of magnetic field lines. Furthermore, letting $\gamma \rightarrow \infty$ yields exactly (4.4), and the instability criterion for the incompressibility case can be recovered from the compressible case as $\gamma \rightarrow \infty$.

(3) Magnetic field is parallel to the gravitational field $B_{0} \| \vec{g}$ for incompressible fluids:

$$
B_{0}=\left(B_{0}, 0,0\right) .
$$

In this case, we consider a density profile $\rho_{0}$ whose gradient has a negative average over $(0,2 \pi)$, which is stronger than (4.4):

$$
\int_{0}^{2 \pi} \rho_{0 x}(x) d x<0 .
$$

The linear operator $\mathcal{L}$, the Sturm-Liouville equation, and $\lambda_{k}$ are given by:

$$
\begin{aligned}
& \mathcal{L}(v):=B_{0}^{2} v_{x x}-\nabla\left(p_{t}+B_{0} \cdot Q\right)-\vec{g} \rho_{0 x} v_{1}, \\
& \rho_{0} \lambda_{k}^{2} \tilde{v}_{1}=B_{0}^{2} \tilde{v}_{1 x x}+\frac{\lambda_{k}^{2}}{k^{2}}\left(\rho_{0} \tilde{v}_{1 x}\right)_{x}-\frac{B_{0}^{2}}{k^{2}} \tilde{v}_{1 x x x x}-g \rho_{0 x} \tilde{v}_{1}, \\
& \lambda_{k}^{2}=\sup _{u \in H^{2}(0,2 \pi)} \frac{\int_{0}^{2 \pi} \mathcal{L}(u) \cdot u d x}{\int_{0}^{2 \pi} \rho_{0}\left[u^{2}+\frac{u_{x}^{2}}{k^{2}}\right] d x}>0,
\end{aligned}
$$


where

$$
\begin{aligned}
\mathcal{L}(u) \cdot u & =-F\left(-\frac{u_{x x}}{k}\right)+H\left(u, u_{x}\right), \\
F\left(-\frac{u_{x x}}{k}\right) & =B_{0}^{2} \frac{u_{x x}^{2}}{k^{2}}, H\left(u, u_{x}\right)=-g \rho_{0 x} u^{2}-B_{0}^{2} u_{x}^{2} .
\end{aligned}
$$

$\Lambda$ has the following formula:

$$
\Lambda^{2}=\sup _{v \in H^{1}(D)} \frac{\iint_{D} H\left(v, v_{x}\right) d x d y}{\iint_{D} \rho_{0} v^{2} d x d y}>0 .
$$

Lemma 4. $\Lambda^{2}$ in (4.14) is a positive real number.

Proof. We can take a family of test periodic functions in $H^{1}$ that guarantees the positivity of $\Lambda^{2}$. Let $c=\min _{x} \rho_{0}(x)$ and

$$
\phi^{n}(x, y)=c-\frac{x}{n} \text { for } 0 \leq x<2 \pi .
$$

Since

$$
\begin{aligned}
& \iint_{D}-g \rho_{0 x}\left(\phi^{n}\right)^{2} d x d y \rightarrow c^{2} \iint_{D}-g \rho_{0 x} d x d y>0 \text { as } n \rightarrow \infty, \\
& B_{0}^{2} \iint_{D}\left(\phi_{x}^{n}\right)^{2} d x d y=\frac{4 \pi^{2} B_{0}^{2}}{n^{2}} \rightarrow 0 \text { as } n \rightarrow \infty
\end{aligned}
$$

we can choose large $n$ to get the positivity of the numerator of $\Lambda^{2}$.

(4) Magnetic field is parallel to the gravitational field $B_{0} \| \vec{g}$ for compressible fluids:

$$
\begin{aligned}
\mathcal{L}(v) & =B_{0}^{2} v_{x x}-\nabla\left(B_{0} \cdot Q\right)-\nabla\left(p_{0 x} v_{1}+\gamma p_{0} \nabla \cdot v\right)-\vec{g}\left(\rho_{0 x} v_{1}+\rho_{0} \nabla \cdot v\right), \\
\lambda_{k}^{2}= & \sup _{\substack{u=\left(u_{1}, u_{2}\right) \\
u_{1} \in H^{1}(0,2 \pi), u_{2} \in H^{1}(0,2 \pi)}} \frac{\int_{0}^{2 \pi} \mathcal{L}(u) \cdot u d x}{\int_{0}^{2 \pi} \rho_{0}\left[u_{1}^{2}+u_{2}^{2}\right] d x}<0,
\end{aligned}
$$

where

$$
\begin{aligned}
\mathcal{L}(u) \cdot u & =-F\left(u_{2 x}\right)-G\left(u_{1}, u_{1 x}, u_{2}\right)+H\left(u_{1}, u_{1 x}\right), \\
F\left(u_{2 x}\right) & =B_{0}^{2} u_{2 x}^{2}, \\
G\left(u_{1}, u_{1 x}, u_{2}\right) & =\left(\gamma p_{0}+B_{0}^{2}\right)\left[\frac{\gamma p_{0}}{\gamma p_{0}+B_{0}^{2}} u_{1 x}+\frac{g \rho_{0} u_{1}}{\gamma p_{0}+B_{0}^{2}}+k u_{2}\right]^{2}, \\
H\left(u_{1}, u_{1 x}\right) & =\frac{1}{\gamma p_{0}+B_{0}^{2}}\left[g \rho_{0} u_{1}-B_{0}^{2} u_{1 x}\right]^{2}-g \rho_{0 x} u_{1}^{2}-B_{0}^{2} u_{1 x}^{2},
\end{aligned}
$$

and

$$
\Lambda^{2}=\sup _{v \in H^{1}(D)} \frac{\iint_{D} H\left(v, v_{x}\right) d x d y}{\iint_{D} \rho_{0} v^{2} d x d y}>0 .
$$

The instability condition is given by

$$
\int_{0}^{2 \pi}\left(\frac{g^{2} \rho_{0}^{2}}{\gamma \rho_{0}+B_{0}^{2}}-g \rho_{0 x}\right) d x>0 .
$$


5. Energy estimate and approximate solution. In this section, we construct approximate solutions using a method in [8] originated by Grenier, and we provide energy estimates for the fully nonlinear MHD system for both incompressible and compressible fluids.

We first construct approximate solutions. In the construction, $\delta>0$ denotes an arbitrary small parameter, and $\theta$ is a small but fixed positive constant (independent of $\delta$ ). We choose and fix $k_{0}$ with $\lambda=\lambda_{k_{0}}$ (dominant eigenvalue) such that

$$
\Lambda<2 \lambda \text {. }
$$

We define $T^{\delta}$ by

$$
\theta=\delta \exp \left(\lambda T^{\delta}\right)
$$

Equivalently,

$$
T^{\delta}=\frac{1}{\lambda} \ln \frac{\theta}{\delta}
$$

We rewrite the full system (1.6) in the vector form for

$$
\begin{aligned}
& w=(\sigma(t, x, y), v(t, x, y), p(t, x, y), B(t, x, y)): \\
& w+A^{1}(w) \partial_{x} w+A^{2}(w) \partial_{y} w+\mathcal{L}(w)=F(w) .
\end{aligned}
$$

An approximate solution $w^{a}(t, x, y)=\left(\sigma^{a}(t, x, y), v^{a}(t, x, y), p^{a}(t, x, y), B^{a}(t, x, y)\right)$ is of the form

$$
w^{a}(t, x, y)=\sum_{j=1}^{N} \delta^{j} r_{j}(t, x, y),
$$

such that

$$
w^{a}+A^{1}\left(w^{a}\right) \partial_{x} w^{a}+A^{2}\left(w^{a}\right) \partial_{y} w^{a}+\mathcal{L}\left(w^{a}\right)=F\left(w^{a}\right)+R_{N}^{a} .
$$

We show the existence of such approximate solutions in the following lemma. The key point is that we can choose a dominant eigenvalue $\lambda$ with $\Lambda<2 \lambda$ to make this construction work.

Lemma 5. For any fixed integer $N>0$, there exists an approximate solution (5.3) satisfying (5.4). Furthermore, for every integer $s \geq 0$, there is small $\theta>0$ such that if $0 \leq t \leq T^{\delta}$ as in (5.2), then the $j$-th coefficient $r_{j}$ and the remainder $R_{N}^{a}$ satisfy

$$
\begin{aligned}
\left\|r_{j}\right\|_{H^{s}} & \leq C(s, N) \exp (j \lambda t), \text { for } 1 \leq j \leq N, \\
\left\|R_{N}^{a}\right\|_{H^{s}} & \leq C(s, N) \delta^{N+1} \exp \{(N+1) \lambda t\} .
\end{aligned}
$$

Proof. The construction of $r_{j}$ will be made by induction on $j$. The idea is as follows. We split the system into linear and nonlinear parts:

$$
\begin{aligned}
& \partial_{t} w^{a}+A^{1}(0) \partial_{x} w^{a}+A^{2}(0) \partial_{y} w^{a}+\mathcal{L}\left(w^{a}\right) \\
& =\left[A^{1}\left(w^{a}\right)-A^{1}(0)\right] \partial_{x} w^{a}+\left[A^{2}\left(u^{a}\right)-A^{2}(0)\right] \partial_{y} w^{a}-F\left(w^{a}\right)=: h(\delta)
\end{aligned}
$$

with the Taylor expansion of $w^{a}$ in $\delta$

$$
w^{a}=\sum_{j=1}^{N} \delta^{j} r_{j} .
$$


Thus, $r_{j}$ is the solution of the part of (5.7) that corresponds to the coefficient of $\delta^{j}$ in its Taylor expansion.

For $j=1$, take for $r_{1}$ the smooth normal growing mode to the linearized system with our chosen wave number $k_{0}$ and the corresponding dominant eigenvalue $\lambda=\lambda_{k_{0}}$ as in (2.2). Clearly this growing mode fulfills (5.5).

Assuming that we have constructed $r_{j}(j<N)$ that satisfies (5.5), we construct $r_{j+1}$. Let

$$
u_{j}=\sum_{k=1}^{j} \delta^{k} r_{k}(t, x, y) .
$$

Define the nonlinear part of the system substituted by $u_{j}$ as

$$
h_{j+1}(\delta)=\left[A^{1}\left(u_{j}\right)-A^{1}(0)\right] \partial_{x} u_{j}+\left[A^{2}\left(u_{j}\right)-A^{2}(0)\right] \partial_{y} u_{j}-F\left(u_{j}\right) .
$$

Since this is the nonlinear part of the system and the terms in $\delta^{j+1}$ come from the terms $\delta^{k}$ for $1 \leq k \leq j$, it is enough to consider $u_{j}$ in order to collect the $(j+1)$-th coefficient of the nonlinear part of the expansion. Then $r_{j+1}$ is defined to be a solution of

$$
\begin{aligned}
& \partial_{t} r_{j+1}+A^{1}(0) \partial_{x} r_{j+1}+A^{2}(0) \partial_{y} r_{j+1}+\mathcal{L}\left(r_{j+1}\right) \\
& =\frac{-h_{j+1}^{(j+1)}(0)}{(j+1) !}
\end{aligned}
$$

with initial data $r_{j+1}(0, x, y)=0$. Notice that

$$
\frac{-h_{j+1}^{(j+1)}(0)}{(j+1) !}=\sum_{j_{1} m_{1}+j_{2} m_{2}+\ldots+j_{p+1} m_{p+1}=j+1} B_{J, i}^{M} r_{j_{1}}^{m_{1}} r_{j_{2}}^{m_{2}} \cdots r_{j_{p}}^{m_{p}} \partial_{i} r_{j_{p+1}}^{m_{p+1}},
$$

where $m_{k} \geq 0,1 \leq k \leq j$, and $B_{J, i}^{M}$ depends on $A^{i}$ and $F$. The induction hypothesis (5.5) for $r_{k}, 1 \leq k \leq j$ applies to obtain, for all $s$,

$$
\begin{aligned}
\left\|\frac{h_{j+1}^{(j+1)}(0)}{(j+1) !}\right\|_{H^{s}} & \leq C(s, N) \exp \left[\left(j_{1} m_{1}+j_{2} m_{2}+\ldots+j_{p+1} m_{p+1}\right) \lambda t\right] \\
& =C(s, N) \exp [(j+1) \lambda t] .
\end{aligned}
$$

By the linear estimates for $\Lambda$ in Lemma 4 and the Duhamel principle, we have

$$
\begin{aligned}
\left\|r_{j+1}(t, \cdot)\right\|_{H^{s}} & \leq C \int_{0}^{t} e^{\Lambda(t-\tau)}\left\|\frac{h_{j+1}^{(j+1)}(0)}{(j+1) !}(\tau)\right\|_{H^{s+2}} \\
& \leq C(s, N) \int_{0}^{t} e^{\Lambda(t-\tau)} e^{(j+1) \lambda \tau} d \tau \\
& \leq C(s, N) e^{(j+1) \lambda t},
\end{aligned}
$$

since $j+1 \geq 2$ and $\Lambda<2 \lambda$.

We now define $w^{a}=\sum_{j=1}^{N} \delta^{j} r_{j}$, and it satisfies

$$
\partial_{t} w^{a}+A^{1}(0) \partial_{x} w^{a}+A^{2} \partial_{y} w^{a}+\mathcal{L}\left(w^{a}\right)=-\sum_{j=1}^{N} \frac{\delta^{j} h_{j}^{(j)}(0)}{j !} .
$$


Then $R_{N}^{a}$ is defined to be the sum of all higher terms than $N$ in the nonlinear part of the $\delta$-expansion (5.7):

$$
R_{N}^{a}=h(\delta)-\sum_{j=1}^{N} \frac{\delta^{j} h_{j}^{(j)}(0)}{j !},
$$

which clearly satisfies (5.6) and the proof is complete.

We state the local-in-time existence for the incompressible ideal MHD equajtions:

Lemma 6 (Local existence to the full system). For all $s \geq 3$ and for any given initial data $\left(\sigma_{0}, v_{0}, p_{0}, B_{0}\right) \in\left(H^{s}(D)\right)^{8}$ such that $\rho(0) \equiv \rho_{0}(x)+\sigma_{0}(x, y) \geq m>0$, there is a $T>0$ such that there exists a unique solution $(\sigma, v, p, B) \in C\left([0, T]:\left(H^{s}(D)\right)^{8}\right)$ to (1.6) with $\rho(t)=\rho_{0}(x)+\sigma(t, x, y)>0$.

We first treat the incompressibility case and we use the vector identities (1.11)-(1.12) to get:

$$
\begin{gathered}
\sigma_{t}+v \cdot \nabla\left(\rho_{0}+\sigma\right)=0, \\
\left(\rho_{0}+\sigma\right)\left(v_{t}+v \cdot \nabla v\right)=-\nabla\left(p+\frac{1}{2} B^{2}+B_{0} \cdot B\right)+B \cdot \nabla B \\
+B_{0} \cdot \nabla B+B \cdot \nabla B_{0}+\sigma \vec{g}, \\
B_{t}=B_{0} \cdot \nabla v-v \cdot \nabla B_{0}+B \cdot \nabla v-v \cdot \nabla B,
\end{gathered}
$$

where $\hat{k}$ is the unit vector in the $z$ direction. Let

$$
w(t, x, y)=(\sigma(t, x, y), v(t, x, y), p(t, x, y), B(t, x, y)) \in C\left([0, T] ;\left(H^{s}(D)\right)^{8}\right)
$$

be a local-in-time solution as constructed above.

Let $w^{a}(t, x, y)=\left(\sigma^{a}(t, x, y), v^{a}(t, x, y), p^{a}(t, x, y), B^{a}(t, x, y)\right)$ be an approximate solution. We now estimate the difference $w^{d}(t, x, y)=w(t, x, y)-w^{a}(t, x, y)$ :

$$
\begin{aligned}
& \sigma_{t}^{d}+\nabla \sigma^{d} \cdot v+\nabla\left(\sigma^{a}+\rho_{0}\right) \cdot v^{d}=-R_{N, 1}^{a}, \\
& \left(\rho_{0}+\sigma\right)\left(v_{t}^{d}+v^{d} \cdot \nabla v+v^{a} \cdot \nabla v^{d}\right)+\sigma^{d}\left(v_{t}^{a}+v^{a} \cdot \nabla v^{a}\right) \\
& =-\nabla\left(p^{d}+\frac{1}{2} B^{d}\left(B+B^{a}\right)+B_{0} \cdot B^{d}\right)+B^{d} \cdot \nabla B+B^{a} \cdot \nabla B^{d} \\
& +B_{0} \cdot \nabla B^{d}+B^{d} \cdot \nabla B_{0}+\sigma^{d} \vec{g}-R_{N, 2}^{a}, \\
& B_{t}^{d}=B_{0} \cdot \nabla v^{d}-v^{d} \cdot \nabla B_{0}+B^{d} \cdot \nabla v+B^{a} \cdot \nabla v^{d}-v^{d} \cdot \nabla B-v^{a} \cdot \nabla B^{d}-R_{N, 3}^{a} .
\end{aligned}
$$

Lemma 7. Let $s \geq 3$ be an integer and assume $\|\sigma\|_{\infty} \leq \frac{1}{2}\left\|\rho_{0}\right\|_{\infty}$. Then there exists a continuous positive function $C$ depending only on $s, \rho_{0}$ such that

$$
\begin{aligned}
\frac{d}{d t}\left\|w^{d}\right\|_{s}^{2} & \leq C\left(\left\|w^{d}\right\|_{s},\left\|w^{a}\right\|_{s+1}\right)\left\|w^{d}\right\|_{s}^{2} \\
& +\left\|R_{N, 1}^{a}\right\|_{s}^{2}+\left\|R_{N, 2}^{a}\right\|_{s}^{2}+\left\|R_{N, 3}^{a}\right\|_{s}^{2} .
\end{aligned}
$$

Proof. This energy estimate is straightforward and thus we give a brief sketch. We take $\partial_{\alpha}$ of the equations (5.8)-(5.10), multiply them by $\partial_{\alpha} w$, and integrate them over $D$. The symmetric terms such as $\nabla \partial_{\alpha} \sigma^{d} \cdot v$ in the first equation vanish upon integration due to 
the divergence-free condition for $v, v^{a}, v^{d}, B, B^{a}, B^{d}$. We now estimate the nonsymmetric terms. Terms that need attention are $\left(B^{d} \cdot \nabla\right) \partial_{\alpha} B^{d},\left(B^{a} \cdot \nabla\right) \partial_{\alpha} B^{d},\left(B_{0} \cdot \nabla\right) \partial_{\alpha} B^{d}$ from the $v$-equation (5.9) and $\left(B^{d} \cdot \nabla\right) \partial_{\alpha} v^{d},\left(B^{a} \cdot \nabla\right) \partial_{\alpha} v^{d},\left(B_{0} \cdot \nabla\right) \partial_{\alpha} B^{d}$ from the $B$ equation (5.10). Upon integration, the three corresponding pairs exactly cancel out. To see this, by the divergence-free condition for $v$ and $B$, we have

$$
\begin{aligned}
& B_{i}^{d} \partial_{i} \partial_{\alpha} B_{j}^{d} \partial_{\alpha} v_{j}^{d}=\partial_{i} B_{i}^{d} \partial_{\alpha} B_{j}^{d} \partial_{\alpha} v_{j}^{d}-B_{i}^{d} \partial_{i} \partial_{\alpha} v_{j}^{d} \partial_{\alpha} B_{j}^{d}=-B_{i}^{d} \partial_{i} \partial_{\alpha} v_{j}^{d} \partial_{\alpha} B_{j}^{d}, \\
& B_{i}^{a} \partial_{i} \partial_{\alpha} B_{j}^{d} \partial_{\alpha} v_{j}^{d}=\partial_{i} B_{i}^{a} \partial_{\alpha} B_{j}^{d} \partial_{\alpha} v_{j}^{d}-B_{i}^{a} \partial_{i} \partial_{\alpha} v_{j}^{d} \partial_{\alpha} B_{j}^{d}=-B_{i}^{a} \partial_{i} \partial_{\alpha} v_{j}^{d} \partial_{\alpha} B_{j}^{d}, \\
& B_{0 i} \partial_{i} \partial_{\alpha} B_{j}^{d} \partial_{\alpha} v_{j}^{d}=\partial_{i} B_{0 i} \partial_{\alpha} B_{j}^{d} \partial_{\alpha} v_{j}^{d}-B_{0 i} \partial_{i} \partial_{\alpha} v_{j}^{d} \partial_{\alpha} B_{j}^{d}=-B_{0 i} \partial_{i} \partial_{\alpha} v_{j}^{d} \partial_{\alpha} B_{j}^{d} .
\end{aligned}
$$

Therefore we obtain the lemma.

We now extend the energy estimates to the compressibility case using a symmetrizer, which is necessary in this compressibility case. We write the full system (1.6) in components:

$$
\begin{aligned}
& \sigma_{, t}+v_{i} \sigma_{, i}+\left(\rho_{0}+\sigma\right) v_{i, i}+\rho_{0 x} v_{1}=0, \\
& \left(\rho_{0}+\sigma\right)\left\{v_{j, t}+v_{i} v_{j, i}\right\}+\left(\rho_{0}+\sigma\right) q \sigma_{, j}+B_{i} B_{i, j}-B_{i} B_{j, i}+B_{0} B_{3, j} \\
= & {\left[\rho_{0} q\left(\rho_{0}\right)-\left(\rho_{0}+\sigma\right) q\right] \rho_{0, j}+B_{i} B_{0, i}-B_{0, j} B_{3}, } \\
& B_{j, t}-B_{i} v_{j, i}+v_{i, i} B_{j}+v_{i} B_{j, i}+v_{i, i} B_{0}=-v_{i} B_{0, i} .
\end{aligned}
$$

Here $j=1,2,3$ and $i$ means the sum over $i=1,2,3$ twice.

We rewrite the full system near the steady state $\left(\rho_{0}, \overrightarrow{0}, B\right)$ in the vector notations for $w=\left(\sigma, v_{1}, v_{2}, v_{3}, B_{1}, B_{2}, B_{3}\right)$ :

$$
w_{t}+A^{1}(w) \partial_{x} w+A^{2}(w) \partial_{y} w+\mathcal{L}(w)=F(w) .
$$

Introducing and multiplying the symmetrizer with $q(\rho)=\gamma \rho^{\gamma-2}$,

$$
D=\operatorname{diag}\left(q\left(\rho_{0}+\sigma\right), \rho_{0}+\sigma, \rho_{0}+\sigma, \rho_{0}+\sigma, 1,1,1\right),
$$


yields the following symmetric matrices:

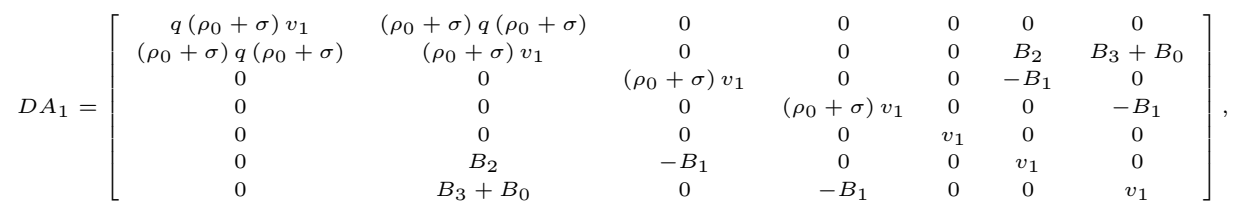

$$
\begin{aligned}
& D A_{2}=\left[\begin{array}{ccccccc}
q\left(\rho_{0}+\sigma\right) v_{2} & 0 & \left(\rho_{0}+\sigma\right) q\left(\rho_{0}+\sigma\right) & 0 & 0 & 0 & 0 \\
0 & \left(\rho_{0}+\sigma\right) v_{2} & 0 & 0 & -B_{2} & 0 & 0 \\
\left(\rho_{0}+\sigma\right) q\left(\rho_{0}+\sigma\right) & 0 & \left(\rho_{0}+\sigma\right) v_{2} & 0 & B_{1} & 0 & B_{3}+B_{0} \\
0 & 0 & 0 & \left(\rho_{0}+\sigma\right) v_{2} & 0 & 0 & -B_{2} \\
0 & -B_{2} & B_{1} & 0 & v_{2} & 0 & 0 \\
0 & 0 & 0 & 0 & 0 & v_{2} & 0 \\
0 & 0 & B_{3}+B_{0} & -B_{2} & 0 & 0 & v_{2}
\end{array}\right], \\
& D A_{3}=\left[\begin{array}{ccccccc}
q\left(\rho_{0}+\sigma\right) v_{3} & 0 & 0 & \left(\rho_{0}+\sigma\right) q\left(\rho_{0}+\sigma\right) & 0 & 0 & 0 \\
0 & \left(\rho_{0}+\sigma\right) v_{3} & 0 & 0 & -B_{3} & 0 & 0 \\
0 & 0 & \left(\rho_{0}+\sigma\right) v_{3} & 0 & 0 & -B_{3} & 0 \\
\left(\rho_{0}+\sigma\right) q\left(\rho_{0}+\sigma\right) & 0 & 0 & \left(\rho_{0}+\sigma\right) v_{3} & B_{1} & B_{2} & B_{0} \\
0 & -B_{3} & 0 & B_{1} & v_{3} & 0 & 0 \\
0 & 0 & -B_{3} & B_{2} & 0 & v_{3} & 0 \\
0 & 0 & 0 & B_{0} & 0 & 0 & v_{3}
\end{array}\right] \text {, } \\
& D \mathcal{L}(u)=\left[\begin{array}{c}
\rho_{0 x} q\left(\rho_{0}\right) v_{1} \\
q\left(\rho_{0}\right) \rho_{0 x} \sigma+\rho_{0} q^{\prime}\left(\rho_{0}\right) \rho_{0 x} \sigma+B_{0 x} B_{3} \\
0 \\
-B_{0 x} B_{1} \\
0 \\
0 \\
B_{0 x} v_{1}
\end{array}\right] \\
& D F(u)=\left[\begin{array}{c}
\rho_{0 x} q\left(\rho_{0}\right) v_{1}-\rho_{0 x} q\left(\rho_{0}+\sigma\right) v_{1} \\
{\left[\left(\sigma+\rho_{0}\right) q\left(\sigma+\rho_{0}\right)-\rho_{0} q\left(\rho_{0}\right)-q\left(\rho_{0}\right) \rho_{0 x} \sigma-\rho_{0} q^{\prime}\left(\rho_{0}\right) \rho_{0 x} \sigma\right] \rho_{0 x}} \\
0 \\
0 \\
0 \\
0 \\
0
\end{array}\right] .
\end{aligned}
$$

Now that $D A^{1}, D A^{2}$, and $D A^{3}$ are symmetric, we have the following local-in-time solution to the full system via standard energy estimates for a symmetrizable hyperbolic system as in [3], 15] in the absence of magnetic fields.

LEMmA 8. For all integers $s \geq 3$ and for any given initial data $\left(\sigma_{0}, v_{0}, p_{0}, B_{0}\right) \in\left(H^{s}(D)\right)^{8}$ such that $\rho(0) \equiv \rho_{0}(x)+\sigma_{0}(x, y) \geq m>0$, there is a $T>0$ such that there exists a unique solution $u=(\sigma, v, p, B) \in C\left([0, T]:\left(H^{s}(D)\right)^{8}\right)$ to (1.6) with $\rho(t)=\rho_{0}(x)+$ $\sigma(t, x, y)>0$.

Since the construction of approximate solutions is similar to the incompressibility case as in Lemma 5 for a hyperbolic system, we omit it. We now estimate the difference of an exact solution and an approximate solution. Let

$$
w(t, x, y)=(\sigma(t, x, y), v(t, x, y), B(t, x, y)) \in C\left([0, T]: H^{s}(D)\right)
$$

be an exact solution and $w^{a}(t, x, y)=\left(\sigma^{a}(t, x, y), v^{a}(t, x, y), B^{a}(t, x, y)\right)$ be an approximate solution as constructed in Lemma 5 . Then their difference is

$$
w^{d}=w-w^{a}=\left(\sigma-\sigma^{a}, v-v^{a}, B-B^{a}\right),
$$


and it satisfies

$$
\begin{aligned}
& w_{t}^{d}+\sum_{i=1}^{3} A^{i}\left(w^{a}+w^{d}\right) \partial_{i} w^{d}+\sum_{i=1}^{3}\left[A^{1}\left(w^{a}+w^{d}\right)-A^{1}\left(w^{a}\right)\right] \partial_{x} w^{a} \\
= & -\mathcal{L}\left(w^{d}\right)+F(w)-F\left(w^{a}\right)-R_{N}^{a} .
\end{aligned}
$$

This symmetrizable hyperbolic system for $w^{d}$ allows the following energy estimates. The proof is straightforward by classical energy methods as in [3, 8].

Lemma 9. For any integer $s \geq 3$, there exists a continuous function $g_{s}(\cdot, \cdot)$ such that

$$
\frac{\partial}{\partial t}\left\||| w^{d}||_{s}^{2} \leq g_{s}\left(\left.\left|\left\|w^{d}\left|\left\|_{s},||\left|w^{a}\right|\right\|_{s+1}\right)\right\|\right|\left|w^{d}\right|\right|_{s} ^{2}+\left|\left\|R_{N}^{a} \mid\right\|_{s}^{2},\right.\right.\right.
$$

where $\left.\||\cdot|\|\right|_{s}$ is defined by

$$
\||| v||_{s}^{2}=\sum_{|\alpha| \leq s} \partial_{\alpha} v D\left(\rho_{0}+\sigma\right) \partial_{\alpha} v
$$

Here $D$ is the symmetrizer in (5.12).

Notice that $\||\cdot|\|_{s}$ is related to the usual norm $\|\cdot\|_{H^{s}}$ by

$$
\eta\|v\|_{H^{s}} \leq\|\| v\|\|_{s} \leq C_{s}\left(\|\sigma\|_{H^{s}},\left\|\rho_{0}\right\|_{H^{s}}\right)\|v\|_{H^{s}},
$$

since $\rho_{0} \geq c>0$ and so $D \geq \eta I d$ for some $\eta>0$ and $s \geq 3$.

Notice that all three norms $\|\cdot\|_{s},\||\cdot|\|_{s}$, and $\|\cdot\|_{H^{s}}$ are equivalent since $\rho_{0}$ is smooth with a positive minimum.

\section{Nonlinear instability.}

Proof of Theorem 2. Let $w^{a}(t, x, y)=\left(\sigma^{a}(t, x, y), v^{a}(t, x, y), p^{a}(t, x, y), B^{a}(t, x, y)\right)$ be an approximate solution with $N$ to be determined later. For any $\delta>0$, there exists a local-in-time solution $w^{\delta}(t, x, y)=\left(\sigma^{\delta}(t, x, y), v^{\delta}(t, x, y), p^{\delta}(t, x, y), B^{\delta}(t, x, y)\right)$ with the initial data $w^{a}(0)$ to the full system (1.6). By Lemma 7 and Lemma 9, we have

$$
\frac{d}{d t}\left\|w^{d}\right\|_{s}^{2} \leq C\left(\left\|w^{d}\right\|_{s},\left\|w^{a}\right\|_{s+1}\right)\left\|w^{d}\right\|_{s}^{2}+C \delta^{2(N+1)} e^{2(N+1) \lambda t}
$$

with $w^{d}(0)=0$.

Let

$$
T=\sup \left\{t \mid\left\|w^{a}\right\|_{s+1} \leq \omega / 2,\left\|w^{d}\right\|_{s} \leq \omega / 2\right\},
$$

where $\omega$ is a small positive number that assures the local existence. Then $T$ (depending on $\delta$ ) is well defined since $w^{d}(0)=0$ and $\left\|w^{\alpha}(0)\right\|_{s+1}=O(\delta)$. We claim that the instability time $T^{\delta}$ occurs within the existence time $T$, that is, $T^{\delta} \leq T$. Suppose not, i.e., $T<T^{\delta}$. Then for $t \leq T$, by our construction of approximate solutions, we have

$$
\left\|w^{a}\right\|_{s+1} \leq C \sum_{j=1}^{N} \delta^{j}\left\|r_{j}(t)\right\|_{H^{s}} \leq \sum_{j=1}^{N} C_{j} \delta^{j} e^{j \lambda t} \leq \sum_{j=1}^{N} C_{j} \theta^{j}<\omega / 2,
$$

if $\theta$ is small. Now we appeal to the definition of $T$ and (6.1) to get, for $t \leq T$,

$$
\frac{d}{d t}\left\|w^{d}\right\|_{s}^{2} \leq C(\omega / 2, \omega / 2)\left\|w^{d}\right\|_{s}^{2}+C \delta^{2(N+1)} e^{2(N+1) \lambda t} .
$$


We choose $N>0$ satisfying

$$
C(\omega / 2, \omega / 2)<2(N+1),
$$

so that $\left\|w^{d}\right\|_{s}$ has a growth rate at most $(N+1) \lambda$. Then using the Gronwall inequality yields

$$
\left\|w^{d}\right\|_{s} \leq C \delta^{2(N+1)} e^{2(N+1) \lambda t}=C \theta^{2(N+1)}<\omega / 2,
$$

if $\omega$ is small. Thus we can deduce $T^{\delta} \leq T$. Now at the instability time $T^{\delta}$, we have

$$
\begin{aligned}
\left\|w^{a}\left(T^{\delta}\right)\right\|_{L^{1}} & \geq \delta\left\|r_{1}\left(T^{\delta}\right)\right\|_{L^{1}}-\sum_{j=2}^{N} \delta^{j}\left\|r_{j}\left(T^{\delta}\right)\right\|_{L^{1}} \\
& \geq C \delta e^{\lambda T^{\delta}}-\sum_{j=2}^{N} C_{j} \delta^{j} e^{j \lambda T^{\delta}} \\
& =C \theta-\sum_{j=2}^{N} C_{j} \theta^{j} \geq \frac{C}{2} \theta .
\end{aligned}
$$

We then deduce at time $T^{\delta}$,

$$
\begin{aligned}
\left\|w^{\delta}\left(T^{\delta}\right)\right\|_{L^{1}} & \geq\left\|w^{\delta}\left(T^{\delta}\right)\right\|_{L^{1}}-\left\|\left(w^{\delta}-w^{a}\right)\left(T^{\delta}\right)\right\|_{L^{1}} \\
& \geq\left\|w^{a}\left(T^{\delta}\right)\right\|_{L^{1}}-\left\|\left(w^{\delta}-w^{a}\right)\left(T^{\delta}\right)\right\|_{H^{s}} \\
& \geq \frac{C}{2} \theta-C \theta^{N+1} \geq \frac{C}{4} \theta=\varepsilon_{0}>0 .
\end{aligned}
$$

This completes the proof.

Acknowledgement. This research is supported by Postech Research Fund 2006.

\section{REFERENCES}

[1] G. Bateman, MHD instabilities, MIT Press, Cambridge, MA, 1980.

[2] I. B. Bernstein, E. A. Frieman, M. D. Kruskal, R. M. Kulsrud, An energy principle for hydromagnetic stability problems, Proc. Roy. Soc. (London) A244 (1958), 17-40. MR0091737 (19:1009e)

[3] S. Cordier, E. Grenier, Y. Guo, Two-stream instabilities in plasmas, In honor of C. S. Morawetz. Methods Appl. Anal. 7 (2000), no. 2, 391-405. MR1869291 (2003g:82091)

[4] S. Chandrasekhar, Hydrodynamic and hydromagnetic stability, The International Series of Monographs on Physics, Clarendon Press, Oxford 1961. MR.0128226 (23:B1270)

[5] S. Chandrasekhar, The stability of the radiative gradients in the interior of a star, Proc. Natl. Acad. Sci. U. S. 23 (1937), 572-577.

[6] C. Cherfils-Cléouin, O. Lafitte, P-A. Raviart, Asymptotic results for the linear stage of the RayleighTaylor instability, Mathematical fluid mechanics, 47-71, Adv. Math. Fluid Mech., Birkhäuser, Basel, 2001. MR1865049 (2002h:76059)

[7] T. G. Cowling, The stability of gaseous stars, Monthly Notices Roy. Astro. Soc. 94 (1934), 768.

[8] E. Grenier, Semiclassical limit of the nonlinear Schrödinger equations, Proc. AMS. 126 (1998), no. 2, 523-530. MR 1425123 (98d:35204)

[9] Y. Guo, W. Strauss, Instability of periodic BGK equilibria, Comm. Pure. Appl. Math. 48 (1995), 861-894. MR 1361017 (96j:35252)

[10] H. J. Hwang, Y. Guo, On the dynamical Rayleigh-Taylor instability, Arch. Rational Mech. Anal. 167 (2003), 235-253. MR1978583 (2004f:76064)

[11] H. Jeffreys, The stability of a layer of fluid heated below, Phil. Mag. 2 (1926), 833-844.

[12] M. D. Kruskal, M. Schwarzschild, Some instabilities of a completely ionized plasma, Proc. Roy. Soc. (London) A223 (1954), 348-360. MR0061997(15:914a) 
[13] P-L. Lions, Mathematical topics in fluid mechanics. Vol. 2. Compressible models. Oxford Lecture Series in Mathematics and its Applications, 3. Oxford Science Publications. The Clarendon Press, Oxford University Press, New York, 1996. MR1637634 (99m:76001)

[14] L. D. Landau, E. M. Lifshitz, Fluid mechanics, 2nd ed., Pergamon, New York, 1987. MR961259 (89i:00006)

[15] A. Majda, Compressible fluid flows and systems of conservation laws in several variables. Appl. Math. Sci. 53, Springer, 1984.

[16] D. Nicholson, Introduction to plasma theory, John Wiley \& Sons, 1983.

[17] E. R. Priest, Solar magnetohydrodynamics, London, 1983.

[18] L. Rayleigh, Analytic solutions of the Rayleigh equation for linear density profiles, Proc. London. Math. Soc. 14 (1883), 170-177.

[19] K. Schwarzchild, Equilibrium of the sun's atmosphere, Nachr. Kgl. Ges. Wiss. Göttingen 1 (1906), 41-53.

[20] Yu. A. Tserkovikov, Convective instability of a rarefied plasma. Doklady Akad. Nauk S. S. S. R. 130 (1960), 295 [translation: Soviet Phys.-Doklady 5 (1960), 87]. 